\title{
Conciencia emocional, estados de ánimo e indicadores de ajuste individual y social en niños de 8-12 años
}

\author{
${ }^{1}$ Departamento de Psicología, Universitat Jaume I de Castellón \\ ${ }^{2}$ Universidad Europea de Valencia \\ ${ }^{3}$ Departamento de Psicología, Universitat de Valencia
}

Lidón Villanueva $^{1 *}$, Vicente Prado-Gascó ${ }^{2}$, Remedios González ${ }^{3}$ e Inmaculada Montoya ${ }^{3}$

\begin{abstract}
Resumen: La conciencia emocional o experiencia consciente de la emoción es un concepto clave relacionado con diferentes indicadores de ajuste individual del menor, como las quejas somáticas. Esta relación, mediatizada por los estados de ánimo, se ha demostrado en población preadolescente y adolescente. Sin embargo, apenas se ha analizado en franjas de edad más bajas e incluyendo otros indicadores de ajuste a diferentes contextos, como la adaptación. Por ello, el objetivo de este trabajo es el de analizar la contribución de la conciencia emocional y los estados de ánimo, sobre diferentes indicadores de ajuste individual (quejas somáticas) y de adaptación (evaluada a través del test TAMAI), en niños de 8-12 años. Para ello, se administraron diferentes cuestionarios de autoinforme a 1423 menores con una media de 9.8 años. Los resultados apoyan la influencia de la conciencia emocional (principalmente de la habilidad de diferenciar y comunicar verbalmente las emociones y analizar las propias emociones), reforzada por los estados de ánimo, no sólo sobre las quejas somáticas, sino sobre la inadaptación personal y social. Todo ello subraya la importancia, tanto de las habilidades emocionales como de los estados afectivos que las acompañan, en la vida cotidiana del menor.

Palabras clave: Conciencia emocional; estados de ánimo; quejas somáticas;
\end{abstract} adaptación; diferenciar emociones; conciencia corporal.

\section{Introducción}

La experiencia emocional es heterogénea, ya que el ser humano puede ser consciente de ella o por el contrario, no ser consciente. Esta experiencia consciente de las emociones, la conciencia emocional, contribuye al desarrollo personal y al bienestar (Bisquerra y Pérez, 2007), a una regulación emocional adaptativa, así como a aumentar nuestro autoconocimiento (Lambie, 2009; Lambie y Marcel, 2002). Dado que la reflexión consciente sobre la experiencia emocional permite al niño interrumpir el programa emocional en marcha y ganar en flexibilidad, adaptándose a la nueva situación, la baja conciencia emocional puede verse reflejada en niños con conducta agresiva, con síntomas depresivos, etc. (Stegge y Meerum Terwogt, 2007), o bien disminuir la utilización de estrategias adaptativas de regulación cognitiva, por ejemplo, en casos de enfermedad crónica (Lahaye, Fantini-Hauwel, Van Broeck, Bodart y Luminet, 2011b).

Incluida como una de las competencias emocionales clave, la conciencia emocional es definida por Rieffe et al., (2008b), como un proceso atencional que sirve para monitorizar y diferenciar las emociones, localizar sus antecedentes, e incluso ignorar la activación física que forma parte de la experiencia emocional, como la tensión en el área abdominal. La conciencia emocional incluye también aspectos actitudinales, tales como la forma en que las experiencias y ex-

* Dirección para correspondencia [Correspondence address]: Lidón Villanueva, Universitat Jaume I, Avda. Sos Baynat, s/n, 12071, Castellón (España). E-mail: bvillanu@psi.uji.es
Title: Emotional awareness, mood and individual and social adjustment outcomes in children aged 8-12 years old.

Abstract: Emotional awareness or conscious experience of emotion is a key concept related to different individual adjustment child outcomes, such as somatic complaints. This relationship, moderated by mood, has been found in preadolescent and adolescent population. However, little is known in younger children and including other adjustment outcomes, such as adaptation. Therefore, the objective of this work is to analyze the contribution of emotional awareness and mood upon different adjustment outcomes: somatic complaints and inadaptation (assessed by the test TAMAI), in children aged 8-12 years old. Different self-reported questionnaires were administered to 1423 children, with a mean age of 9.8 years old. Results support the influence of emotional awareness (mainly the ability to differentiate and verbally communicate emotions and analyze the own emotions), strengthened by mood, not only upon somatic complaints, but also upon personal and social inadaptation. These results come to outline the importance of emotional abilities and the corresponding affective moods, in children daily life.

Key words: Emotional awareness; mood; somatic complaints; adaptation; differentiating emotions; bodily awareness.

presiones emocionales son detectadas en uno mismo y en los demás.

En ocasiones, algunos conceptos relacionados se han asimilado al término de competencia emocional, principalmente, el de inteligencia emocional y el de alexitimia. El primero de estos términos, la inteligencia emocional, se refiere a la identificación, comprensión, expresión, regulación y uso de las emociones propias y ajenas (Mayer y Salovey, 1997). En este sentido, resulta excesivamente amplio, ya que incluye dimensiones que no forman parte de las competencias emocionales, o que incluso son consideradas como resultados de las mismas, como por ejemplo, el bienestar del menor (Lahaye et al., 2011a; Lahaye, Luminet, Van Broeck, Bodart y Mikolajczak, 2010). Algunos de los aspectos de la conciencia emocional que forman parte de la inteligencia emocional rasgo son: la expresión emocional, la diferenciación de emociones y el tener en cuenta las emociones de otros (Rieffe et al., 2008b). Por su parte, el término alexitimia es demasiado restrictivo, ya que alude a problemas con la identificación y descripción de los propios sentimientos (Rieffe, Oosterveld y Meerum Terwogt, 2006), no incluyendo, sin embargo, la regulación de las mismas o el análisis de las emociones de los demás. Esta conceptualización de la alexitimia muestra cierto solapamiento conceptual con la parte atencional de la conciencia emocional (diferenciar emociones, compartirlas verbalmente, etc.), (Rieffe, Villanueva, Adrián y Górriz, 2009).

Algunos estudios han recogido la influencia de la conciencia emocional en diversos planos del desarrollo infantil, como los problemas internalizantes (ansiedad, depresión, 
etc.) (Rieffe et al., 2008b; Rieffe y De Rooij, 2012), las quejas somáticas (Jellesma, Rieffe, Meerum Terwogt y Kneepkens, 2006; Rieffe et al., 2009; van der Veek, Nobel y Derkx, 2012b), la competencia social (Mavroveli, Petrides, Rieffe y Bakker, 2008), o la adaptación académica y social (Mestre, Guil, Lopes, Salovey y Gil, 2006). Sin embargo, pocos estudios han integrado, en un mismo trabajo, diversos indicadores infantiles de funcionamiento adecuado, aunando tanto el plano individual como la adaptación a diferentes contextos. Por lo tanto, el objetivo de este trabajo sería el de analizar la contribución de la conciencia emocional infantil a diversos resultados del menor, en concreto, las quejas somáticas y los problemas de adaptación.

En el plano individual puede encontrarse una de las relaciones más estrechas y probadas que presenta la conciencia emocional: su conexión con la salud del menor, reflejada en las quejas somáticas autoinformadas. No obstante, esta relación parece encontrarse mediatizada por los estados de ánimo. Los estados de ánimo son considerados como estados afectivos difusos o globales, que aparecen sin una causa aparente. Su intensidad por lo general es baja, aunque pueden durar horas o incluso días, como la tristeza, la alegría, etc. (Scherer, 2005). En concreto, la relación planteada entre las tres variables sería la siguiente: el hecho de presentar escasas habilidades de competencia emocional impide a la persona tratar la situación emocional de forma adaptativa. De esta forma, es probable la aparición de estados de ánimo negativos (como la tristeza, el enfado, el miedo, etc.), que conducen a altos niveles de estrés prolongado, y pueden desembocar finalmente en quejas somáticas (Rieffe, Meerum Terwogt y Jellesma, 2008a; Rieffe et al., 2010).

Esta relación entre quejas somáticas, conciencia emocional y estados de ánimo ha sido confirmada en población preadolescente y adolescente (Rieffe et al., 2009; Mavroveli et al., 2008; Lahaye et al., 2010). Diversos estudios comprobaron de forma consistente que, especialmente la incapacidad para diferenciar entre diferentes emociones, y una fuerte conciencia de síntomas corporales durante la experiencia emocional, contribuían de forma independiente a la predicción de quejas somáticas. Asimismo, una falta de predisposición para atender a las propias emociones también predecía una mayor frecuencia de quejas somáticas (Rieffe et al., 2008a), mientras que un aumento de la atención a las emociones de los demás, predecía más quejas somáticas en adolescentes (Rieffe et al., 2007).

No obstante, son muy escasos los estudios que han analizado la relación entre conciencia emocional, estados de ánimo y quejas somáticas, en población de menor edad. Por ejemplo, Rieffe y De Rooij (2012) observaron que fueron las habilidades de diferenciar emociones, y la conciencia corporal las más relacionadas con diferentes síntomas internalizantes, como las quejas somáticas. Por su parte, van der Veek, Derkx, de Haan, Benninga y Boer (2012a) observaron diferencias en conciencia emocional en niños de 7-18 años con dolores abdominales funcionales y sin dolores, aunque el tamaño de estas diferencias fue pequeño. Sin embargo, los propios autores concluyen que el diseño de su estudio no permite la investigación de procesos evolutivos, aconsejando un análisis que contemple con más detalle la variable edad. Además, precisamente en el tramo de edad de la infancia tardía se producen desarrollos emocionales importantes para manejar adecuadamente las emociones negativas. Por ejemplo, sobre los 9 años, los niños se vuelven más hábiles a la hora de reflexionar sobre sus propias emociones (Harris, 1989), o son capaces de aceptar la posibilidad de experimentar simultáneamente emociones muy diferentes y comprenden las reglas que llevan a esconder emociones (Stegge y Meerum Terwogt, 2007). Por ello, este estudio pretende cubrir la franja de edad de los 8-12 años, previa a desarrollos preadolescentes y adolescentes.

En un plano más amplio de los indicadores de ajuste, la conciencia emocional y/o inteligencia emocional se ha relacionado en menor medida con problemas de adaptación del menor en los diferentes entornos: social, escolar, familiar, etc. Como concepto clave que determina el grado de éxito en el proceso educativo y socializador, sí se ha relacionado, entre otros, con la existencia de psicopatología y sintomatología depresiva y ansiosa (Lastra, Martínez-Chamorro y Luzuriaga, 2001), con el rendimiento escolar (Borges, Hernández y González, 2011) o con la presencia de situaciones de crisis familiar (Martín, García y Siverio, 2012). Sin embargo, son escasos los estudios que analizan la relación entre adaptación y plano emocional. En estos estudios, se ha podido comprobar cómo la inteligencia emocional autoevaluada y objetiva correlacionaba negativamente con inadaptación escolar y con inadaptación general del test TAMAI (autoinforme), en población de 14-32 años (Fernández, Iglesias y Barraca, 2007). Con población de menor edad (12-15 años), y medidas autoinformadas de ajuste social y personal, continuaba encontrándose la misma relación (Salguero, Fernández-Berrocal, Ruiz-Aranda, Castillo y Palomera, 2011). Igualmente, cuando las medidas de adaptación escolar y social se obtenían a través de los profesores de los menores adolescentes, la habilidad de inteligencia emocional también correlacionaba positivamente con estas medidas (Mestre et al., 2006). Dado que se ha comprobado que la adaptación en todos los ámbitos de los niños es diferente a la de los adolescentes (García y Siverio, 2005; Siverio y García, 2007), se hace necesario un análisis específico de la relación conciencia emocional-adaptación en este período de edad de la infancia tardía, así como de la contribución de los estados de ánimo a esta relación.

Por todo lo anterior, este estudio pretende analizar la influencia de la conciencia emocional y de los estados de ánimo en distintos indicadores de ajuste individual y de adaptación del menor, en una franja de edad poco estudiada, y a través de medidas de autoinforme. Estas medidas han sido consideradas válidas en niños de 7-8 años, así como de 1112 años, por ejemplo, mostrándose informantes fiables de síntomas físicos comunes (Lundqvist, Rugland, Clench-Aas, Bartonova y Hofoss, 2010). En concreto, las hipótesis que se plantean al respecto son las siguientes: Todos los compo- 
nentes de la conciencia emocional, con la excepción de la habilidad de compartir verbalmente las emociones, y la habilidad de no esconderlas, predecirán las quejas somáticas del menor. Asimismo, la conciencia emocional también contribuirá a predecir los problemas de adaptación del menor. Ambas predicciones mejorarán con la inclusión de los estados de ánimo (tristeza, miedo, enfado y felicidad).

Respecto a la influencia de las variables sexo y edad, en primer lugar, se hipotetiza una mejora madurativa con la edad, en todas las variables analizadas. En segundo lugar, no se espera diferenciación entre el valor predictivo de los distintos componentes de la conciencia emocional en función del sexo. En cambio, en la línea de estudios previos (Abad, Forns, Amador y Martorell, 2000; Leadbeater, Kuperminc, Blatt y Hertzog, 1999; Lozano y García Cueto, 2000), se podría esperar que ciertos estados de ánimo de carácter internalizante (tristeza, miedo) fueran mejores predictores de las variables resultado en chicas que en chicos, en los cuales resultarían mejores predictores los estados de ánimo relacionados con aspectos externalizantes, como por ejemplo, el enfado.

\section{Método}

\section{Participantes}

Los participantes en el estudio fueron un total de 1423 menores de 8-12 años de edad, con una media de 9.8 años $(N=231$ de 8 años; $N=310$ de 9 años; $N=390$ de 10 años; $N=378$ de 11 años; $N=114$ de 12 años). De ellos, 746 eran chicas, lo cual representaba el 52\% del total (677 chicos, $48 \%$ ). Todos ellos eran de nivel socioeconómico medio y procedían de los cursos de $3^{\circ}-6^{\circ}$ de Educación Primaria, de 12 colegios ubicados en 7 localidades de la Comunidad Valenciana.

\section{Instrumentos}

El Cuestionario de Conciencia Emocional (EAQ)(Rieffe et al., 2008b).- Se utilizó una versión de la escala original, adaptada por los autores del presente trabajo. En lugar de los 30 ítems originales, estaba compuesta por 28 ítems, agrupados en 6 factores clave de la conciencia emocional: Diferenciar emociones, Compartir emociones verbalmente, No esconder emociones, Conciencia corporal de las emociones, Tener en cuenta las emociones de los demás, y Análisis de las propias emociones. Los factores contaban con una escala de respuesta tipo Likert de tres anclajes $(1=$ nunca, $2=$ a veces, $3=$ a menudo), y en todos ellos una puntuación elevada significaba una mayor presencia de esa habilidad. Todas las escalas mostraron adecuadas propiedades psicométricas en estudios previos (Rieffe et al., 2008b; Rieffe et al., 2009), lo cual también se confirmó en el caso del presente estudio (Tabla 1). (Índices de Bondad Ajuste con estimación por Máxima verosimilitud $(M L)$ con la corrección robusta de S-B: $p<.001$; $\chi^{2}(d f)=5709.25$ (378); S-B $\chi^{2}(d f)=805.7900$ (335); NFI $=$ $.92, C F I=.91, I F I=.91$, RMSE $A=.03 ; \alpha=.59-.65)$.

El Cuestionario de Estados de Ánimo (Mood; Rieffe, Meerum Terwogt y Bosch, 2004).- Se utilizó una versión del cuestionario adaptada por el equipo investigador (Górriz, Prado-Gascó, Villanueva, Ordóñez y González, 2013), consistente en 4 estados de ánimo: Felicidad, Enfado, Tristeza y Miedo. Se entrenó a los niños para contestar cada ítem en una escala de respuesta de tres puntos $(1=$ nunca, $2=$ a veces, $3=$ a menudo). En todas las dimensiones, puntuaciones mayores implican una mayor presencia de ese estado de ánimo. En estudios anteriores (Rieffe et al., 2009), así como en el actual, las escalas demostraron unas propiedades psicométricas adecuadas. (Índices de Bondad Ajuste con estimación por Máxima verosimilitud $(M L)$ con la corrección robusta de S-B: $p<.001 ; \quad \chi^{2}(d f)=489.68(200) ; \quad$ S-B $\chi^{2}(d f)=402.14(200) ; \mathrm{NNFI}=.92, C F I=.94, I F I=.94$, RMSE $A=.04 ; \alpha=.69-.78)$.

El Listado de Quejas Somáticas (SCL; Rieffe et al., 2006; 2007).- Formado por 11 ítems, adaptados por el equipo investigador, este listado identifica la frecuencia con que los menores experimentan y sienten dolor, como dolor de estómago, de cabeza, etc. Posee una escala de respuesta de tres puntos $(1=$ nunca, $2=$ a veces, $3=$ a menudo), indicando una mayor puntuación, mayor presencia de quejas somáticas. Este listado ha demostrado una buena fiabilidad y validez en estudios previos (Rieffe et al., 2009), así como en el presente estudio (Índices de Bondad Ajuste con estimación por Máxima verosimilitud $(M L)$ con la corrección robusta de S-B: $p$ $<.001 ; \chi^{2}(d f)=157.96(29) ; N F I=.92, C F I=.93, I F I=.93$, RMSE $A=.06 ; \alpha=.81)$.

El Test Autoevaluativo MultifactoriaI de Adaptación Infantil (TAMAI; Hernández, 1998).- Se trata de una escala de evaluación de la inadaptación general, personal, social, escolar, familiar y hacia los hermanos para edades comprendidas entre los 8 y 18 años. Este instrumento posee adecuadas propiedades psicométricas de acuerdo con el autor (Hernández, 1998). El formato de respuesta es dicotómico $(0=$ no, $1=$ si), indicado la presencia o ausencia de cada uno de los enunciados. En todas las dimensiones, mayores puntuaciones implican niveles más elevados de inadaptación $(\alpha=.87)$.

Todos los cuestionarios se trasladaron y adaptaron siguiendo los criterios internacionales de la International Test Commission (ITC) (Hambleton, 2005).

\section{Procedimiento}

Para la obtención de los datos se recurrió a un muestreo de tipo intencional. Los cuestionarios se administraron en 100 aulas de 12 colegios de la Comunidad Valenciana, previa autorización de la Consejería de Educación, el Consejo Escolar de cada centro y los padres de los menores. 
Su administración se llevó a cabo en las aulas de los centros escolares, en horario escolar, durante la mañana, entre los meses de Octubre y Marzo 2010-2011, dependiendo del centro en cuestión. El orden de presentación de los cuestionarios fue contrabalanceado para evitar los efectos de fatiga que se pudieran derivar. Su aplicación fue colectiva, organizada en 2-3 sesiones diferentes de 1 hora aproximadamente, con una extensión máxima de 3 semanas.

\section{Análisis estadístico}

El análisis estadístico de los datos se realizó por medio del paquete estadístico SPSS (versión 20). En primer lugar se calcularon los estadísticos descriptivos más importantes para cada dimensión de los constructos considerados, y las correlaciones de Spearman entre las mismas. Se recurrió a este estadístico puesto que la escala de medida utilizada en dos de los instrumentos se compone de tres anclajes (1 a 3). Seguidamente se procedió al estudio de modelos de regresión lineal jerárquica en los cuales las variables de la conciencia emocional en primer lugar, y junto a los estados de ánimo más tarde, servían como predictores de variables de ajuste (Inadaptación general, personal, social, y Quejas somáticas). Previamente se comprobaron los supuestos necesarios del modelo de regresión jerárquica de los modelos finales (Field, 2005), resultando todos adecuados.

El siguiente paso fue analizar la influencia que la edad y el género tienen sobre los diferentes modelos propuestos; para ello, en primer lugar se procedió a calcular las comparaciones de medias, mediante el estadístico no paramétrico "U" de Mann-Whitney y las correlaciones de Spearman. A continuación se analizaron los modelos de regresión anteriormente realizados en función del género y de la edad. No obstante, en este trabajo tan sólo se presentan los modelos de regresión en función del género, al no realizar la edad ninguna contribución significativa. Tampoco se presentan aquí los análisis realizados con los apartados Inadaptación escolar, familiar y hacia los hermanos, del test TAMAI, debido a su escasa contribución significativa.

\section{Resultados}

Los resultados de los análisis descriptivos de las variables estudiadas se muestran en la Tabla 1 . Los valores obtenidos por las puntuaciones en Conciencia emocional, Estados de ánimo y Quejas somáticas se encuentran alrededor de la media. En lo que respecta a las puntuaciones en Inadaptación, los valores medios se encuentran en un rango bajomoderado para la Inadaptación general y social (centil entre 21-60), y un rango moderado para la Inadaptación personal (centil entre 41-60), dentro de los criterios del test TAMAI.

En segundo lugar, se presentan las correlaciones entre las variables principales (ver Tabla 2). Entre las correlaciones de los factores de Conciencia Emocional destaca el valor moderado de las mismas (de .06* a .39**), así como los valo- res negativos de la Conciencia Corporal con la mayoría de componentes emocionales. Por lo que respecta a las correlaciones entre factores de Estados de Ánimo, éstas presentan valores más elevados que los anteriores (de -.13** a .56**). Por último, las correlaciones entre los factores de ambos constructos mostraron correlaciones significativas positivas entre los componentes emocionales y el estado de Felicidad, y correlaciones significativas negativas con los estados de ánimo negativos. En el caso de la Conciencia Corporal, el patrón encontrado era inverso.

Asimismo, se calcularon las correlaciones Spearman para las variables predictoras y las variables resultados (ver Tabla 3). Las correlaciones entre las subescalas del Cuestionario EAQ con las variables resultados (Quejas, IG, IS y IP) fueron las esperadas (negativas en todos los casos, con la excepción de la Conciencia Corporal). Por su parte, las cuatro subescalas del Cuestionario de Estados de ánimo se relacionaron con las variables resultados también en la dirección prevista (positiva para los estados negativos Enfado, Tristeza y Miedo; negativa para el estado positivo de Felicidad). Las correlaciones de las diferentes variables con la edad presentaron resultados en la línea de estudios previos: una mayor edad estaba asociada con menos Quejas somáticas, menor Inadaptación general y personal, menor Tristeza y Miedo y más Felicidad. A nivel de conciencia emocional, sólo las subescalas Diferenciar emociones y Emociones de los otros correlacionaban positivamente con la edad $\left(r=.12^{* *} ; r=\right.$ $.10^{* *}$, respectivamente), sugiriendo un pequeño pero significativo efecto de la maduración en estas dimensiones, mientras que Comunicar verbalmente lo hacía de forma negativa $\left(r=-.06^{*}\right)$.

Tabla 1. Estadísticos descriptivos: Rango, medias, desviaciones típicas y coeficientes alpha de las variables de estudio.

\begin{tabular}{clllll}
\hline & Variables & Rango & $M$ & $D T$ & $\alpha$ \\
\hline & Dif. & $1-3$ & 2.32 & .44 & .654 \\
& C.V. & $1-3$ & 2.05 & .56 & .586 \\
P. & $1-3$ & 2.36 & .43 & .626 \\
& O. & $1-3$ & 2.58 & .40 & .654 \\
& N.E. & $1-3$ & 2.08 & .53 & .612 \\
C.C. & $1-3$ & 2.14 & .51 & .634 \\
F. & $1-3$ & 2.76 & .35 & .762 \\
F. & E. & $1-3$ & 1.51 & .45 & .778 \\
T. & T. & $1-3$ & 1.35 & .39 & .693 \\
SCL & M. & $1-3$ & 1.51 & .42 & .696 \\
Quejas & $1-33$ & 15.46 & 3.63 & .810 \\
& IG & B-Mod & 26.5 & 13.9 & .874 \\
& IS & B-Mod & 7.53 & 4.57 & .775 \\
& IP & Mod & 10.05 & 5.52 & .816 \\
\hline
\end{tabular}

EAQ: Dif: Diferenciar emociones; C.V.: Comunicar verbalmente; P: Análisis propias emociones; O: Emociones de los otros; N. E.: No esconder emociones; C.C.: Conciencia corporal. Estados de ánimo: T: Tristeza; M: Miedo; E: Enfado; F: Felicidad. TAMAI: IG: Inadaptación General, IS: Inadaptación Social, IP: Inadaptación Personal. B: Bajo. Mod: Moderado.

A continuación, se presentan los análisis de regresión jerárquica con las quejas somáticas (SCL), y las variables de inadaptación (TAMAI) como variables criterio, y Conciencia 
Emocional (EAQ) y Estados de Ánimo (Mood) como variables predictoras. En un primer paso, se incluyeron todas las subescalas del cuestionario Conciencia Emocional, y en el segundo paso, las variables de Estados de Ánimo. En el primer paso, todas las escalas del EAQ (con la excepción de la escala No esconder emociones), predijeron un $17 \%$ de la varianza de las Quejas somáticas $(F=58.53)$. En concreto, fueron las escalas: Diferenciar emociones (Beta tipificado $=$ .24), Comunicar verbalmente (Beta tipificado $=-.14$ ), Análisis propias emociones (Beta tipificado $=-.12$ ), Emociones de los otros (Beta tipificado $=-.09)$, y Conciencia corporal (Beta tipificado $=.16)$.

Tabla 2. Correlaciones Spearman de las subescalas de Conciencia Emocional y Estados de Ánimo.

\begin{tabular}{|c|c|c|c|c|c|c|c|c|c|c|}
\hline & 1 & 2 & 3 & 4 & 5 & 6 & 7 & 8 & 9 & 10 \\
\hline 1. Dif. & - & & & & & & & & & \\
\hline 2. C.V. & $.31^{* *}$ & - & & & & & & & & \\
\hline 3. P. & $-.16^{* *}$ & $.05^{*}$ & - & & & & & & & \\
\hline 4. $\mathrm{O}$. & $.06^{*}$ & $.15^{* *}$ & $.39^{* *}$ & - & & & & & & \\
\hline 5. N.E. & $.26^{* *}$ & $.32^{* *}$ & -.04 & $.13^{* *}$ & - & & & & & \\
\hline 6. C.C. & $-.29^{* *}$ & $-.17^{* *}$ & $.26^{* *}$ & $.14^{* *}$ & $-.16^{* *}$ & - & & & & \\
\hline 7. F. & $.10^{* *}$ & $.14^{* *}$ & $.23^{* *}$ & $.23^{* *}$ & $.06^{*}$ & .03 & - & & & \\
\hline 8. E. & $-.20^{* *}$ & $-.20^{* *}$ & $-.07^{* *}$ & $-.14^{* *}$ & $-.17^{* *}$ & $.14^{* *}$ & $-.19^{* *}$ & - & & \\
\hline 9. T. & $-.26^{* *}$ & $-.26^{* *}$ & $-.06^{*}$ & $-.18^{* *}$ & $-.17^{* *}$ & $.13^{* *}$ & $-.29^{* *}$ & $.56^{* *}$ & - & \\
\hline 10. M. & $-.25^{* *}$ & $-.24^{* *}$ & -.01 & $-.08^{* *}$ & $-.11^{* *}$ & $.21^{* *}$ & $-.13^{* *}$ & $.46^{* *}$ & $.53^{* *}$ & - \\
\hline
\end{tabular}

EAQ: Dif: Diferenciar emociones; C.V.: Comunicar verbalmente; P: Análisis propias emociones; O: Emociones de los otros; N. E.: No esconder emociones; C.C.: Conciencia corporal. Estados de ánimo: T: Tristeza; M: Miedo; E: Enfado; F: Felicidad.

Tabla 3. Correlaciones Spearman de las subescalas de Conciencia Emocional, Estados de Ánimo y Edad, con las variables dependientes del estudio.

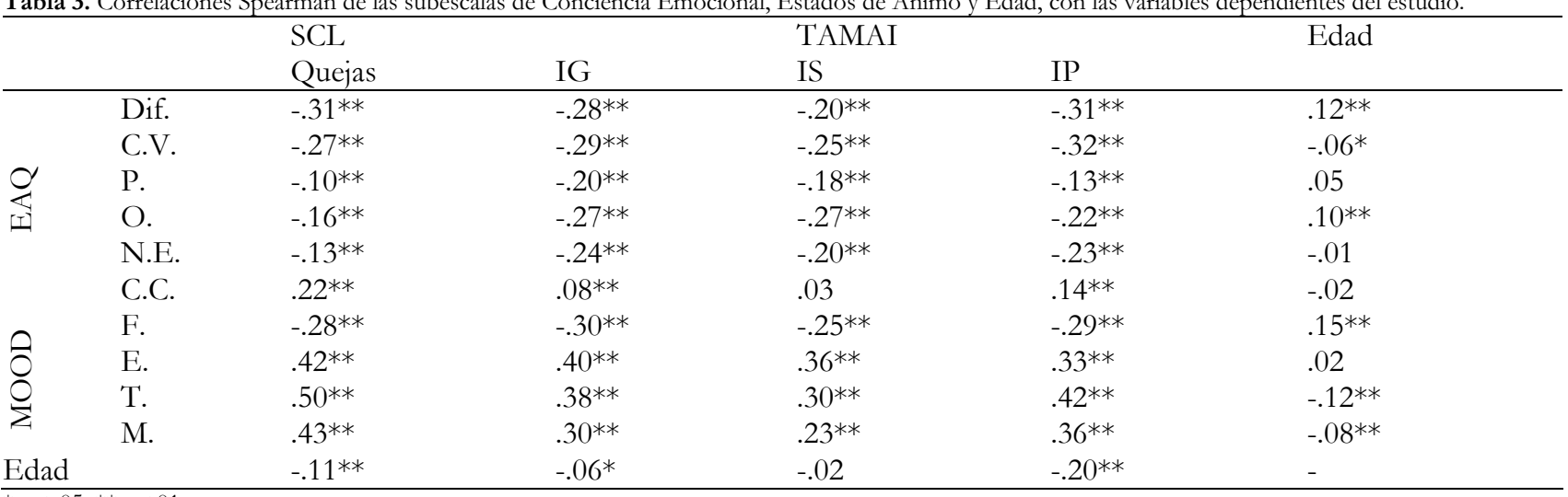

$* p<.05 ; * * p<.01$

EAQ: Dif: Diferenciar emociones; C.V.: Comunicar verbalmente; P: Análisis propias emociones; O: Emociones de los otros; N. E.: No esconder emociones; C.C.: Conciencia corporal. Estados de ánimo: T: Tristeza; M: Miedo; E: Enfado; F: Felicidad. TAMAI: IG: Inadaptación General, IS: Inadaptación Social, IP: Inadaptación Personal.

Respecto a las medidas de Inadaptación, todas las escalas del EAQ (a excepción de la Conciencia corporal) predijeron la Inadaptación general en un $23 \%(F=80.92)$, (Diferenciar Beta tipificado $=-.21$; Comunicar verbalmente Beta tipificado $=$ -.16; Análisis propias emociones Beta tipificado = -.18; Emociones de los otros Beta tipificado $=-.17$; $\mathrm{y}$ No esconder emociones Beta tipificado $=-.10)$. En un análisis más específico de la Inadaptación, todas las escalas del EAQ contribuyeron a la predicción de la Inadaptación personal $\left(\mathrm{R}^{2}\right.$ adj $=.22, F=$ 64.99; Diferenciar emociones (Beta tipificado $=-.21$ ), Comunicar verbalmente (Beta tipificado = -.18), Análisis propias emociones (Beta tipificado $=-.15$ ), Emociones de los demás (Beta tipificado $=-.13)$, Conciencia corporal (Beta tipificado $=$ .10), y No esconder (Beta tipificado $=-.08$ ). Y en cuanto a la Inadaptación social, cuatro escalas del EAQ (con la excepción de No esconder emociones y Conciencia Corporal) la predijeron en un $17 \%(F=68.65)$. Sus contribuciones significativas fueron las siguientes: Diferenciar emociones (Beta tipificado = -.14), Comunicar verbalmente (Beta tipificado $=$ .17), Análisis propias emociones (Beta tipificado = -.21), $\mathrm{y}$ Emociones de los otros (Beta tipificado $=-.13$ ).

El siguiente paso fue añadir las cuatro dimensiones del Cuestionario de Estados de ánimo (Enfado, Tristeza, Miedo y Felicidad), como variables predictoras, además del Cuestionario EAQ (ver tabla 4). Con la adición de los Estados de ánimo a la variable Conciencia emocional, el modelo mejoró sensiblemente. El incremento en $\mathrm{R}^{2}$ fue de .22 en el caso de Quejas somáticas, y de .12 a .15 en el caso de las medidas de Inadaptación. Por su parte, la varianza total explicada aumentó, oscilando entre un $28 \%$ para la Inadaptación social y un 38\% para las Quejas somáticas. En el caso de las Quejas, todas las subescalas contribuyeron a su predicción, con la excepción de No esconder emociones, y Emociones de los otros. En el caso de las variables de Inadaptación, sólo las subescalas de Conciencia corporal y Tristeza no realizaron contribuciones a su predicción. 
Tabla 4. Análisis de regresión para las subescalas del EAQ y Estados de ánimo sobre las quejas somáticas y tres medidas de Inadaptación (General, Personal y Social).

\begin{tabular}{|c|c|c|c|c|c|c|c|c|c|}
\hline \multirow{2}{*}{\multicolumn{2}{|c|}{ Predictores }} & \multirow{2}{*}{\multicolumn{2}{|c|}{$\begin{array}{c}\text { SCL } \\
\beta\end{array}$}} & \multicolumn{2}{|r|}{$\mathrm{IG}$} & \multicolumn{2}{|c|}{ IP } & \multicolumn{2}{|c|}{ IS } \\
\hline & & & & $\Delta R^{2}$ & $\beta$ & $\overline{\Delta R^{2}}$ & $\beta$ & $\overline{\Delta R^{2}}$ & $\beta$ \\
\hline \multirow[t]{3}{*}{$\overline{P a s o}$} & & $.17 * * *$ & & $.23^{* *}$ & & $.22 * * *$ & & $.17 * * *$ & \\
\hline & Dif. & & $-.13^{* * *}$ & & $-.13 * * *$ & & $-.13 * * *$ & & $-.07 * *$ \\
\hline & C.V. & & $-.05^{* *}$ & & $-.09 * * *$ & & $-.11 * * *$ & & $-.09 * * *$ \\
\hline \multirow{4}{*}{ 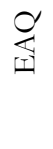 } & P. & & $-.07 * *$ & & $-.13 * * *$ & & $-.09 * * *$ & & $-.08 * *$ \\
\hline & O. & & -.03 & & $-.13 * * *$ & & $-.09 * * *$ & & $-.17 * * *$ \\
\hline & N.E. & & .02 & & $-.08 * *$ & & $-.07 * *$ & & $-.07 * *$ \\
\hline & C.C. & & $.12^{* * *}$ & & .01 & & $.07 * *$ & & -.02 \\
\hline \multicolumn{2}{|c|}{ Paso 2} & $.22 * * *$ & & $.15^{* *}$ & & $.15^{* * *}$ & & $.12^{* * *}$ & \\
\hline \multirow{6}{*}{ 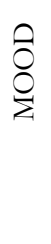 } & F. & & $-.16 * * *$ & & $-.17 * * *$ & & $-.19 * * *$ & & $-.15^{* * *}$ \\
\hline & E. & & $.12^{* * *}$ & & $.21 * * *$ & & $.06^{* *}$ & & $.23^{* * *}$ \\
\hline & $\mathrm{T}$. & & $.23^{* * *}$ & & $.09 * *$ & & $.15^{* * *}$ & & .04 \\
\hline & M. & & $.17 * * *$ & & $.09 * *$ & & $.17 * *$ & & $.07 * *$ \\
\hline & $\begin{array}{l}\text { Total } \\
\mathrm{R}^{2} \mathrm{adj}\end{array}$ & $.38^{* * *}$ & & $.37 * *$ & & $.36^{* * *}$ & & $.28^{* * *}$ & \\
\hline & $\mathrm{n}$ & 1407 & & 1408 & & 1408 & & 1408 & \\
\hline
\end{tabular}

EAQ: Dif: Diferenciar emociones; C.V.: Comunicar verbalmente; P: Análisis propias emociones; O: Emociones de los otros; N. E.: No esconder emociones; C.C.: Conciencia corporal. Estados de ánimo: T: Tristeza; M: Miedo; E: Enfado; F: Felicidad. TAMAI: IG: Inadaptación General, IS: Inadaptación Social, IP: Inadaptación Personal.

Posteriormente se analizó el posible efecto mediador de los Estados de Ánimo sobre la predicción de la Inadaptación y de las Quejas somáticas por parte de la Conciencia Emocional. Los modelos de regresión resultante explicaban menos del 10\% de la varianza, por lo que el efecto mediador no ha podido ser demostrado.

En lo referente a las comparaciones de medias según el género, se pudo comprobar que las chicas muestran mayores puntuaciones en Conciencia corporal, Emociones de los otros, Propias emociones, Miedo y Quejas somáticas frente a los chicos $(U=-6.79, p<.000 ; U=-5.81, p<.000 ; U=-$ 2.67, $p=.008 ; U=-3.91, p<.000 ; U=-2.82, p<.005)$. Por el contrario, son los chicos los que presentan mayor Inadaptación social y general $(U=-4.16, p<.000 ; U=-4.16, p$ $<.000$, respectivamente).

Cuando se realizaron correlaciones en función del género, el patrón fue consistente con las expectativas así como con los resultados de las correlaciones comentadas anteriormente. Las subescalas de Conciencia emocional presentaban correlaciones significativas negativas con las variables de Inadaptación y Quejas somáticas tanto en niños como en niñas: rango de $-.12^{* *}$ a $-.35^{* *}$ (con la excepción de la Conciencia Corporal, de signo positivo: rango de $.12^{* *}$ a $.23^{* *}$ ). Cabe destacar que las subescalas Diferenciar emociones, Comunicarlas verbalmente, No esconderlas y Conciencia corporal (aspectos atencionales) presentaban correlaciones de mayor tamaño en las niñas que en los niños. Mientras, en el caso de Análisis de las propias emociones y Emociones de los otros (aspectos actitudinales), se producía el efecto contrario. Por su parte, las variables Estados de ánimo presentaban valores más altos que los anteriores respecto a las variables de ajuste, de signo positivo en el caso de la Tristeza, Enfado y Miedo (rango de $.24 * *$ a $.52^{* *}$ ), y de signo negativo en el caso de la Felicidad (rango de $-.28^{* *}$ a $-.41^{* *}$ ), pero sin diferencias fundamentales entre niños y niñas.
Por último, se realizaron análisis de regresión jerárquica esta vez en función del género. De nuevo, en un primer paso se incluyeron las subescalas de Conciencia Emocional y en segundo lugar, las subescalas de Estados de Ánimo. Considerando la inclusión de los Estados de Ánimo en el segundo paso, se produjo un incremento en $\mathrm{R}^{2}$ de .19 y .25 para chicos y chicas respectivamente (quejas somáticas), e incrementos que oscilaban entre .11 y .17 para las chicas y .12 y .14 para los chicos, en el caso de los problemas de adaptación. En cuanto al total de varianza explicada, la predicción de Quejas somáticas fue la más alta de todas ellas (42\% y 34\% en chicas y chicos, respectivamente). En el caso de los problemas de adaptación, el rango osciló entre $26 \%$ y $41 \%$ de la varianza. En general, tanto las subescalas de Conciencia Emocional como las de Estados de Ánimo mostraron ser mejores predictoras en el caso de las chicas que en el de los chicos (salvo en Inadaptación social).

\section{Discusión}

El objetivo de este estudio era el de estudiar la influencia de la conciencia emocional y de los estados de ánimo en distintas variables de ajuste de la vida del menor de 8-12 años: quejas somáticas e inadaptación general, social y personal. Tal como se esperaba, los datos obtenidos apoyan parcialmente la primera hipótesis planteada. Es decir, todos los componentes de la Conciencia emocional predecían las Quejas somáticas del menor, con la excepción de Atender las emociones de los demás, y No esconderlas. Mientras que la subescala No esconder emociones es un componente no predictivo clásico, cabe destacar la aparición de un nuevo componente no predictivo de las quejas somáticas, que aparece en esta franja de edad: Atender las emociones de los demás. En cambio, este componente sí aparece como signi- 
ficativo en población preadolescente (Rieffe y De Rooij, 2012), quizás por la importancia de los procesos de comparación social en la formación de la identidad adolescente. Por su parte, el componente Comunicar verbalmente, planteado inicialmente como no predictor de quejas somáticas, en cambio, sí parece predecirlas.

Por lo tanto, el resto de subescalas: Diferenciar emociones, Análisis de las propias emociones, Comunicarlas verbalmente y Conciencia corporal predecían las quejas somáticas, tanto en la muestra total como en los análisis con la variable sexo. La importancia de estos componentes se ve apoyada por estudios previos (Lahaye et al., 2011b; van der Veek et al., 2012b). En el caso de las tres primeras escalas, la relación era negativa, presentando los menores con más quejas somáticas, problemas para diferenciar entre emociones, analizar las suyas propias y comunicarlas, es decir, su conciencia emocional, más que específica, era global. Mientras, en el caso de la Conciencia corporal, la relación era positiva, presentando los niños con alta conciencia corporal, un mayor informe de quejas somáticas. Las posibles explicaciones a esta relación pueden ser diversas; por un lado, los niños con más quejas somáticas, pueden estar centrándose en exceso en los síntomas corporales de la emoción y descuidando el análisis de las causas de la misma. Por otro lado, los niños con quejas somáticas, por lo general presentan emociones más intensas, que se suelen acompañar de signos corporales más salientes (Rieffe et al., 2007).

La segunda hipótesis planteaba que todos los componentes de la conciencia emocional contribuirían a predecir los problemas de adaptación del menor. Esta afirmación se ha visto sustentada por los datos de forma parcial ya que la subescala Conciencia Corporal no aparecía sistemáticamente como variable predictora de las medidas de inadaptación, con la excepción de la inadaptación personal de las chicas. Esta sensibilidad para percibir la relación entre síntoma corporal y emoción (más afín a la problemática de las quejas somáticas), no parece ser tan importante en la predicción de la inadaptación, aunque sí lo es en el caso de las chicas, quienes tradicionalmente han sido consideradas más expresivas y conscientes emocionalmente que los chicos, así como con una mayor presencia de quejas somáticas (Abad et al., 2000).

En tercer lugar, como también se hipotetizaba, la inclusión de los estados de ánimo (junto a la conciencia emocional) ha mejorado la predicción de variables de ajuste individual y adaptación. Todos los estados de ánimo han contribuido a la predicción de quejas somáticas, confirmando estudios previos (Rieffe et al. 2010), mientras que la tristeza ha resultado el estado de ánimo que menor contribución significativa ha realizado en la mayoría de medidas de inadaptación. Los resultados muestran que la conciencia emocional presentaba una limitada contribución independiente en la predicción de quejas somáticas. Parece ser que los efectos de la relación conciencia emocional y variables de ajuste del menor, están moderados por los estados de ánimo. Esto reforzaría la hipótesis de que la conciencia emocional precede a la aparición de los estados de ánimo, los cuales guardan a su vez relación con la percepción de salud que posee el menor. Por lo tanto, centrarse únicamente en la relación directa entre conciencia emocional y quejas somáticas sería una visión reduccionista, tal como plantean van der Veek et al. (2012b). De hecho, cada vez son más numerosos los trabajos que empiezan a plantear relaciones indirectas o variables mediadoras entre la conciencia emocional y la salud del menor, como el sentido de coherencia (Jellesma et al., 2006), las estrategias de afrontamiento (Lahaye et al., 2011b), la ansiedad y depresión (van der Veek et al., 2012b), etc. En este trabajo no se pudo demostrar el efecto mediado, ya que la conciencia emocional no resultó ser un buen predictor de los estados de ánimo. No obstante, sí se comprobó cómo mejoran los modelos de predicción de quejas somáticas e inadaptación, con la inclusión de los estados de ánimo.

En cuarto lugar, se han podido confirmar parcialmente las hipótesis planteadas en relación al sexo y a la edad. En primer lugar, las correlaciones de las diferentes variables con la edad se produjeron en la línea esperada: una mayor edad estaba asociada con menos quejas somáticas, menor inadaptación general y personal, menor tristeza y miedo y más felicidad. Estos resultados son congruentes con trabajos previos (Rieffe et al., 2010; Siverio y García, 2007), en donde, en la franja de edad preadolescente y adolescente, se continuaba mostrando una disminución de las quejas somáticas, pero en cambio, existía una mayor tristeza e inadaptación que en los niños más pequeños, quizás fruto de los cambios vitales y sociales asociados a este período. Otra posible explicación al respecto podría ser que las alteraciones emocionales y conductuales estén menos organizadas en niños pequeños (Lastra et al., 2001), o que incluso estos tengan mayor capacidad para abstraerse de los acontecimientos negativos o para aprender de ellos (García y Siverio, 2005). Respecto a los componentes de la conciencia emocional, sólo la habilidad de diferenciar emociones y atender emociones de los demás progresaban significativamente con la edad. Estos resultados son consistentes con otros estudios, como el de Layahe et al., 2010, quienes plantean que quizás estas subescalas son más sensibles que otras a factores evolutivos. O quizás algunas competencias, como no esconder las emociones, o el análisis de las propias emociones, se adquieren de forma completa antes de los 8 años de edad. No obstante, en general, los efectos de la edad en la conciencia emocional no fueron muy potentes, en la línea de estudios previos (Lahaye et al., 2010; van der Veek et al., 2012a).

Respecto a la variable sexo, en general no se han encontrado diferencias entre niños y niñas en la predicción realizada por los diferentes componentes de conciencia emocional, apoyando trabajos anteriores (Rieffe et al., 2010; Rieffe y De Rooij, 2012). No obstante, las variables de Conciencia Emocional y las de Estados de Ánimo mostraron ser mejores predictoras de las variables resultados en el caso de las chicas que en el de los chicos.

Por último, como posibles limitaciones de los resultados de este trabajo, podrían destacarse las siguientes. En primer 
lugar, en este estudio se utiliza exclusivamente la medida de autoinforme como valoración de las distintas variables. A favor de esta posible limitación cabe decir que los menores poseen un conocimiento directo de sus propios estados internos, siendo más conveniente preguntarles a ellos mismos (Lundqvist et al., 2010). Además, el cuestionario de Conciencia emocional (EAQ) se ha mostrado ampliamente inmune a los efectos de deseabilidad social (Lahaye et al., 2010), rasgo que apoya su validez como cuestionario de autoinforme. A pesar de todo ello, sería necesaria la inclusión de otro tipo de medidas: de terceros, de iguales, de carácter objetivo, etc., que pudieran complementar la información sobre las variables de ajuste individual y social planteadas. Precisamente otra de las limitaciones planteadas en este trabajo se refiere a la necesidad de inclusión de diferentes agentes socializadores de la emoción, como los padres o los iguales. Los cuestionarios utilizados aquí se refieren fundamentalmente al contexto de los iguales (comunicar verbalmente emociones a los compañeros, atender sus emociones, etc.). Sin embargo, el ambiente familiar no se encuentra explícitamente presente, a pesar de resultar crucial en esta franja de edad.

\section{Referencias}

Abad, J., Forns, M., Amador, J. A. y Martorell, B. (2000). Fiabilidad y validez del Youth Self Report en una muestra de adolescentes. Psicothema, 12, 49-54.

Bisquerra, J. y Pérez, N. (2007). Las competencias emocionales. Educación XXI, 10, 61-82.

Borges, A., Hernández, C. M. y González, M. A. (2011). Achivemente predictors in a secondary student's sample. Qualitaty and Quantity. Doi: 10.1007/s11135-011-9547-5.

Fernández, A., Iglesias, A. I. y Barraca, J. (2007). Adaptación psicológica y social en estudiantes e inteligencia emocional. Comunicación presentada en el $8^{\circ}$ Congreso Virtual de Psiquiatría. Recuperado de http://www.psiquiatria.com/articulos/psiq_general_y_otras_areas/psi qsocial $/ 29466 /$

Field, A. (2005). Discovering statistics using spss. Londres: SAGE publications.

García, M. D. y Siverio, M. A. (2005). La tristeza en niños, adolescentes y adultos: Un análisis comparativo. Infancia y Aprendizaje, 28, 453-469.

Górriz, A. B., Prado-Gascó, V., Villanueva, L., Ordóñez, A. y González, R. (2013). The MOOD questionnaire: Adaptation and validation of the Spanish version. Psicothema, 25, 252-257.

Hambleton, R. (2005). Issues, designs and technical guidelines for adapting test into multiple languages and cultures. En R. Hambleton, P. Merenda y C. Spielberger (Eds.), Adapting educational and psychological tests for crosscultural assessment (pp. 3-38). New Jersey: Lawrence Erlbaum.

Harris, P. L. (1989). Children and emotions. The development of psychological understanding. Cambridge, MA, US: Basil Blackwell.

Hernández, P. (1998). Test autoevaluativo multifactorial de adaptación infantil (TAMAI). Madrid: TEA.

Jellesma, F. C., Rieffe, C., Meerum Terwogt, M., y Kneepkens, C. M. F. (2006). Somatic complaints and health care use in children: Mood, emotion awareness and sense of coherence. Social Science and Medicine, 63, 2640-2648.

Lahaye, M., Fantini-Hauwel, C., Van Broeck, N., Bodart, E. y Luminet, O. (2011b). Emotional competence and quality of life of children with asthma: The mediating effect of coping strategies. Psychology and Health, 26. Doi: 10.1080/08870446.2011.562606.

Lahaye, M., Luminet, O., Van Broeck, N., Bodart, E. y Mikolajczak, M. (2010). Psychometric properties of the Emotion Awareness Question-
Asimismo, este trabajo está basado en datos transversales, lo cual excluye las relaciones causales entre las variables objeto de estudio. Por ello, se hacen necesarios estudios longitudinales como el planteado por Rieffe y De Rooij (2012) con la conciencia emocional como variable principal y las quejas somáticas, para determinar las relaciones causales entre esta variable y diversos indicadores de funcionamiento infantil. A pesar de las limitaciones planteadas, el presente estudio apoya la influencia de la conciencia emocional, reforzada por los estados de ánimo, no sólo sobre las quejas somáticas, sino sobre indicadores nuevos de ajuste, como la inadaptación, en una franja de edad diferente a la planteada hasta entonces. Estos resultados subrayan la necesidad de tener en cuenta tanto las habilidades emocionales como los estados afectivos que las acompañan, en la vida cotidiana del menor.

Agradecimientos.- Agradecemos a los centros escolares participantes toda la colaboración y apoyo prestados. Esta investigación ha sido subvencionada por el Ministerio de Ciencia e Innovación, proyecto PSI2010-18742, así como por el Ministerio de Educación, proyecto EDU2010-21791. naire for children in a French-speaking population. Journal of Personality Assessment, 92, 317-326.

Lahaye, M., Mikolajczak, M., Rieffe, C., Villanueva, L., Van Broeck, N., Bodart, E., y Luminet, O. (2011a). Cross-validation of the Emotion Awareness Questionnaire for children in three populations. Journal of Psychoeducational Assessment, 29, 418-427.

Lambie, J. A. (2009). Emotional experience, rational action and selfknowledge. Emotion Review, 3, 272-280.

Lambie, J. A. y Marcel, A. J. (2002) Consciousness and the varieties of emotional experience: A theoretical framework. Psychological Review, 109, 219259.

Lastra, I., Martínez-Chamorro, M. J. y Luzuriaga, C. (2001). El test autoevaluativo multifactorial de adaptación infantil (TAMAI). ¿Útil en el cribado de psicopatología infanto-juvenil? Informaciones Psiquiátricas, 166. Recuperado http://www.revistahospitalarias.org/info_2001/03_166_04.htm

Leadbeater, B. J., Kuperminc, G. P., Blatt, S. J. y Hertzog, C. (1999). A multivariate model of gender differences in adolescents' internalizing and externalizing problems. Developmental Psychology, 35, 1268-1282.

Lozano, L. y García-Cueto, E. (2000). El rendimiento escolar y los trastornos emocionales y comportamentales. Psicothema, 12, 340-343.

Lundqvist, C., Rugland, E., Clench-Aas, J., Bartonova, A. y Hofoss, D. (2010). Children are reliable reporters of common symptoms: Results from a self-reported symptom diary for primary school children. Acta Paediatrica, 99, 1054-1059.

Martín, E., García, M. D. y Siverio, M. A. (2012). Inadaptación autopercibida de los menores en acogimiento residencial. Anales de Psicologia, 28, 541547.

Mavroveli, S, Petrides, K.V., Rieffe, C. y Bakker, F. (2008). Trait emotional intelligence, psychological well-being, and peer-rated social competence in adolescence. En J. C. Cassady y A. E. Mourad (Eds.), Emotional intelligence: Perspectives on educational and positive psychology (pp. 47-62). New York: Peter Lang Publishing, Inc.

Mayer, J. D. y Salovey, P. (1997). What is emotional intelligence? En P. Salovey y D. Sluyter (Eds), Emotional development and emotional intelligence: Implications for educators (p. 3-31). New York: Basic Books. 
Mestre, J. M., Guil, R., Lopes, P. N., Salovey, P. y Gil, P. (2006). Emotional intelligence and social and academic adaptation to school. Psicothema, 18, 112-117.

Rieffe, C. y De Rooij, M. (2012). The longitudinal relationship between emotion awareness and internalizing symptoms during late childhood. European Child and Adolescent Psychiatry. Doi: 10.1007/s00787-012-0267-8.

Rieffe, C., Meerum Terwogt, M., Petrides, K.V., Cowan, C., Miers, A.C., y Tolland, A. (2007). Psychometric properties of the Emotion Awareness Questionnaire for children. Personality and Individual Differences, 43, 95105

Rieffe, C., Meerum Terwogt, M., y Bosch, J. D. (2004). Emotional awareness and somatic complaints in children. European Journal of Developmental Psychology, 1, 31-47.

Rieffe, C., Meerum Terwogt, M., y Jellesma, F. C. (2008a). Emotional competence and health in children. En J. Denollet, I. Nyklicek y A. Vingerhoets (Eds.), Emotion Regulation: Conceptual and Clinical Issues (pp. 184-201). New York: Springer.

Rieffe, C., Oosterveld, P., Meerum Terwogt, M., Novin, S., Nasiri, H., y Latifian, M. (2010). Relationship between alexithymia, mood and internalizing symptoms in children and young adolescents: Evidence from an Iranian sample. Personality and Individual Differences, 48, 425-430.

Rieffe, C., Oosterveld, P., Miers, A.C., Meerum Terwogt, M., y Ly, V. (2008b). Emotion awareness and internalising symptoms in children and adolescents; the Emotion Awareness Questionnaire revised. Personality and Individual Differences, 45, 756-761.
Rieffe, C., Oosterveld, P., y Meerum Terwogt, M. (2006). An alexithymia questionnaire for children: Factorial and concurrent validation results. Personality and Individual Differences, 40, 123-133.

Rieffe, C., Villanueva L., Adrián, J. E. y Górriz, A. B. (2009). Quejas somáticas, estados de ánimo y conciencia emocional en adolescentes. Psicothe$m a, 3,459-464$.

Salguero, J. M., Fernández-Berrocal, P., Ruiz-Aranda, D., Castillo, R. y Palomera, R. (2011). Inteligencia emocional y ajuste psicosocial en la adolescencia: El papel de la percepción emocional. European Journal of Education and Psychology, 4, 143-152.

Scherer, K.R. (2005). What are emotions? And how can they be measured? Social Science Information, 44, 695-729.

Siverio, M. A. y García, M. D. (2007). Autopercepción de adaptación y tristeza en la infancia: la influencia del género. Anales de Psicología, 23, 41-48.

Stegge, H. y Meerum Terwogt, M. (2007). Awareness and regulation of emotion in typical and non typical development. En J. J. Gross (Ed.), Handbook of emotion regulation (pp. 269-286). New York: Guilford Press.

van der Veek, S. M. C., Derkx, H. H. F., de Haan, E., Benninga, M. A. y Boer, F. (2012a). Emotion awareness and coping in children with functional abdominal pain: A controlled study. Social Science and Medicine, 74, 112-119.

van der Veek, S. M., Nobel, R. A. y Derkx, H. H. F. (2012b). The relationship between emotion awareness and somatic complaints in children and adolescents: Investigating the mediating role of anxiety and depression. Psychology and Health. Doi:10.1080/08870446.2012.685738.

(Articulo recibido: 29-9-2012; revisado: 27-2-2013, aceptado: 13-2-2013) 